\title{
Future Patient Demand for Shoulder Arthroplasty by Younger Patients: National Projections
}

\author{
Eric M. Padegimas MD, Mitchell Maltenfort PhD, Mark D. Lazarus MD, \\ Matthew L. Ramsey MD, Gerald R. Williams MD, Surena Namdari MD, MSc
}

Received: 6 September 2014/Accepted: 24 February 2015/Published online: 11 March 2015

(C) The Association of Bone and Joint Surgeons $\mathbb{R} 2015$

\begin{abstract}
Background The outcomes of shoulder arthroplasties in younger patients (55 years or younger) are not as reliable compared with those of the general population. Greater risk of revision and higher complication rates in younger patients present direct costs to the healthcare system and indirect costs to the patient in terms of quality of life. Previous studies have suggested an increased demand for shoulder arthroplasties overall, but to our knowledge, the demand in younger patients has not been explored.
\end{abstract}

One author certifies that he (MDL), or a member of their immediate family, has or may receive payments or benefits, in any one year, an amount of USD 100,001 to USD 1,000,000, from a commercial entity (Tornier, Amsterdam, The Netherlands) related to this work. One author certifies that he (MLR), or a member of their immediate family, has or may receive payments or benefits, in any one year, an amount of USD 10,000 to USD 100,000, from a commercial entity (Integra Life Sciences, Plainsboro, NJ, USA) related to this work. One author certifies that he (GRW), or a member of their immediate family, has or may receive payments or benefits, in any one year, an amount of USD 100,001 to USD 1,000,000, from a commercial entity (DePuy, Warsaw, IN, USA) related to this work.

All ICMJE Conflict of Interest Forms for authors and Clinical Orthopaedics and Related Research ${ }^{\mathbb{R}}$ editors and board members are on file with the publication and can be viewed on request.

This study was performed at The Rothman Institute-Thomas Jefferson University Hospital, Philadelphia, PA, USA.

E. M. Padegimas

Department of Orthopaedic Surgery, Thomas Jefferson

University Hospital, Philadelphia, PA, USA

M. Maltenfort, M. D. Lazarus, M. L. Ramsey,

G. R. Williams, S. Namdari ( $₫)$

The Rothman Institute-Thomas Jefferson University Hospital, 925 Chestnut Street, 5th Floor, Philadelphia, PA 19107, USA

e-mail: Surena.Namdari@gmail.com
Questions/purposes We asked: (1) What was the demand for shoulder arthroplasties between 2002 and 2011 in the United States for all patients and a specific subpopulation of patients who were 55 years old or younger? (2) How is the demand for shoulder arthroplasties in younger patients projected to change through 2030? (3) How is procedural demand projected to change in younger patients through 2030, and specifically, what can we anticipate in terms of hemiarthroplasty volume compared with that of total shoulder arthroplasty?

Methods We used the National Inpatient Sample database to identify primary shoulder arthroplasties performed between 2002 and 2011. A Poisson regression model was developed using the National Inpatient Sample data and United States Census Bureau projections on future population changes to predict estimated national demand for total shoulder arthroplasties and hemiarthroplasties in all patients and in the subpopulation 55 years old or younger. This model was projected until 2030, with associated 95\% CIs. We then specifically analyzed the projected demand of hemiarthroplasties and compared this with demand for all arthroplasty procedures in the younger patient population.

Results Demand for shoulder arthroplasties in patients 55 years or younger is increasing at a rate of $8.2 \%$ per year (95\% CI, 7.06\%-9.35\%), compared with a growth rate of $12.1 \%$ (95\% CI, 8.35\%-16.02\%) per year for patients older than 55 years. In 2002, $15.9 \%$ (3587 of 22,617 captured in the National Inpatient Sample) of primary shoulder arthroplasties were performed in patients 55 years old or younger. In 2011, the relative size of the younger patient population had decreased to $11.0 \%$ ( 7001 of 63,784$)$ of all recipients of shoulder arthroplasties. The demand for primary shoulder arthroplasties among younger patients is projected to increase by $333.3 \%$ (95\% CI, $257.0 \%-432.5 \%$ ) 
from 2011 to 2030. However, in patients older than 55 years demand is projected to increase by $755.4 \%(95 \%$ CI, 380.7\%-1511.1\%). Therefore, despite the increased predicted demand for shoulder arthroplasties in younger patients, they are predicted to account for only $4 \%$ of all recipients by 2030 . The rate of hemiarthroplasties in patients 55 years or younger showed a $16.5 \%$ decline per year (95\% CI, 16.1\%-17.1\%) from 2002 (53.6\% of all arthroplasties) to 2011 (34.2\% of all arthroplasties). By 2030, hemiarthroplasties are projected to account for only $23.5 \%$ of all shoulder arthroplasties in patients 55 years or younger.

Conclusions The demand for shoulder arthroplasties in younger patients continues to increase in the United States; however, rates of hemiarthroplasties are declining. The demand has substantial implications for future revision arthroplasties, which include the direct healthcare costs of revision arthroplasty, the indirect societal burden of missed productivity owing to time away from work, and the increased burden of the need for qualified surgeons to meet the demand. Despite the increasing rate of arthroplasties performed in younger patients, current and projected demands remain greater for older patients, indicating a disproportionately greater need for shoulder arthroplasties in older patients. This is in contrast to the trends observed in the literature regarding hip and knee arthroplasties that show projected demands to be greater in younger patients. Factors responsible for the difference in demand require further investigation but may be related to changing indications, reported poorer outcomes in younger patients, the increased popularity of reverse shoulder arthroplasties in the elderly, or the evolution of nonarthroplasty options. Level of Evidence Level III, prognostic study.

\section{Introduction}

Demand for shoulder arthroplasties in the United States continues to increase and projections based on the $\mathrm{Na}$ tionwide Inpatient Sample (NIS) suggest this trend is likely to continue [11, 19]. While future demand for shoulder arthroplasties, regardless of patient age, has been examined [11, 19], to our knowledge projection of demand for younger patients (55 years old or younger) has not yet been independently analyzed. This is an important gap in our knowledge because indications for shoulder arthroplasties have been expanded to include younger and more active patients. The younger patient with end-stage shoulder arthritis presents a unique treatment challenge given high expectations for outcomes, long life expectancy, and highdemand lifestyles. As a result, the younger patient has been considered at greater risk for needing revision surgery compared with older patients $[35,36]$. Because of concerns regarding aseptic glenoid loosening [12], hemiarthroplasties traditionally have been performed in younger patients. It is unclear whether improved functional outcomes after total shoulder arthroplasties (TSA) in younger patients have resulted in a proportionate increase in TSAs in this population [5]. We intend to address this gap in the knowledge by using the NIS to project the demand for shoulder arthroplasties for the general population and a younger subpopulation. For the purposes of this study, patients 55 years or younger were defined as the young patient population as this age cut-off has been used more consistently in the literature regarding shoulder arthroplasties to determine a younger patient cohort [4, 5]. Additionally, this group of patients has been shown to be at greater risk for needing revision surgery and having poor results after shoulder arthroplasty [4, 5]. Similar analysis in hip and knee arthroplasties has suggested that demand for arthroplasties in younger patients (defined as 65-years-old or younger in that study) is increasing at an accelerated rate [21].

End-stage glenohumeral arthritis in patients is a difficult clinical problem to treat and little is known regarding the current and future demands of arthroplasty treatment. Therefore, a high-quality projection of future shoulder arthroplasty demand in young patients would be valuable. We therefore asked: (1) What was the demand for shoulder arthroplasties between 2002 and 2011 in the United States for all patients and a specific subpopulation of patients who were 55 years old or younger? (2) How is the demand for shoulder arthroplasties in younger patients projected to change through 2030? (3) How is procedural demand projected to change in younger patients through 2030, specifically hemiarthroplasty compared with anatomic TSA?

\section{Methods}

The NIS dataset was used to identify primary shoulder arthroplasties performed in the United States from January 1, 2002 through December 31, 2011. Surgical procedures were identified in the NIS by the International Classification of Diseases, Ninth Revision, Clinical Manifestation (ICD-9-CM). Codes 81.80 (TSA), 81.81 (hemiarthroplasty), and 81.88 (reverse shoulder arthroplasty) were used to identify all primary shoulder arthroplasties performed during our study period. Statistical trend files released by Healthcare Cost and Utilization Project (HCUP) were used to appropriately adjust for yearly variation in sampling methods [17, 21]. We first retrospectively analyzed procedural volume reflected by the three ICD-9-CM codes. We then analyzed the growth trend in demand for the three procedures from 2002 through 2011 to estimate future rate of shoulder arthroplasties through 2030. 
Table 1. Demographics of the NIS sample

\begin{tabular}{|c|c|c|}
\hline Variable & $\begin{array}{l}\text { Patients } \\
\mathrm{n}=84,206\end{array}$ & Percentage \\
\hline Mean age (years) & 68.8 & \\
\hline Patients $\leq 55$ years old & 10,548 & $12.5 \%$ \\
\hline Patients $>55$ years old & 73,514 & $87.5 \%$ \\
\hline Male sex & 33,458 & $39.7 \%$ \\
\hline \multicolumn{3}{|l|}{ Ethnicity } \\
\hline Caucasian & 56,741 & $67.4 \%$ \\
\hline Black & 2549 & $3.0 \%$ \\
\hline Hispanic & 2400 & $2.9 \%$ \\
\hline Asian & 447 & $0.5 \%$ \\
\hline Native American & 223 & $0.3 \%$ \\
\hline Other/unknown* & 22,069 & $26.2 \%$ \\
\hline \multicolumn{3}{|l|}{ Geographic location } \\
\hline Northeast & 9860 & $11.7 \%$ \\
\hline Midwest & 23,878 & $28.4 \%$ \\
\hline South & 30,585 & $36.3 \%$ \\
\hline West & 19,883 & $23.6 \%$ \\
\hline \multicolumn{3}{|l|}{ Practice size } \\
\hline Small & 13,030 & $15.5 \%$ \\
\hline Medium & 19,536 & $23.2 \%$ \\
\hline Large & 51,180 & $60.8 \%$ \\
\hline
\end{tabular}

NIS $=$ National Inpatient Sample; $*=$ other or unknown listing in NIS database without further clarification available.

Patients were identified by the three ICD-9-CM codes for primary arthroplasty. Patients undergoing revision procedures were excluded as the ICD-9CM code used for shoulder arthroplasty (81.97- revision of joint replacement of upper extremity) is not specific for shoulder arthroplasty. Patients 55 years old or younger were designated as younger patients for our study. The sample from the NIS, which is a representative sample of approximately $20 \%$ of all discharges nationally, contained 84,206 patients (Table 1). These are projected to the entire population by the HCUP.

Poisson regression analysis of the NIS and population data from the United States Census Bureau were used to evaluate trends in shoulder arthroplasty demand from 2002 through 2011 and project future demand for all patients and those we designated as "younger" patients through 2030. The interactions among different demographic and surgical variables were used to predict demand for shoulder arthroplasties through 2030. An overdispersion parameter was generated to account for the possibility that the true variability is greater than predictive variability. This is designed to account for the predicted demographic trends from the United States Census Bureau and the predicted surgical trends from the NIS which may understate true population variability. The total number of patients who will undergo shoulder arthroplasties through 2030, with corresponding $95 \%$ CIs, was estimated by comparing the rates estimated by our model with future population projections by the United States Census Bureau. This analysis then was repeated for patients 55 years or younger. This modeling technique has been shown to be valid in previous analyses examining demand projections in hip and knee arthroplasties [21] and in shoulder and elbow arthroplasties [11].

Microsoft ${ }^{\circledR}$ Excel (2013; Redmond, WA, USA) and R (R Development Core Team [2008]; R Foundation for Statistical Computing; Vienna, Austria) were used for all statistical calculations for our study.

\section{Results}

The first component of our analysis focused on trends of shoulder arthroplasty demand from 2002 to 2011 for all patients and a specific subpopulation of patients 55 years old or younger. There were 427,841 primary shoulder arthroplasties performed in the United States during this period, identified by ICD-9-CM codes $81.80,81.81$, and 81.88 , which included any primary shoulder arthroplasty without distinction by underlying diagnosis. The procedural volume increased annually from 25,180 (8.8 arthroplasties per 100,000 people) in 2002 to 67,189 (21.6 arthroplasties/100,000 people) in 2011. Regarding demand in younger patients, in $2002,15.9 \%$ of primary shoulder arthroplasties were performed in patients 55 years or younger. By the end of 2011, the relative size of the younger patient population had decreased to $11 \%$ of all shoulder arthroplasty recipients.

Next, we used a Poisson regression model to attempt to predict future shoulder arthroplasty demand through 2030 for all patients and a subgroup 55 years old or younger. This model projected that the demand for shoulder arthroplasties in all patients to increase to 184.8 cases per 100,000 people by 2030 (95\% CI, 94.5-363.3) compared with 21.6 per 100,000 people in 2011 . This model also predicted that for patients 55 years old or younger the demand would increase to 10.1 cases per 100,000 people (95\% CI, 7.8-13.04) and for patients older than 55 years, 539.8 cases per 100,000 people (95\% CI, 272.1-1079.9) (Fig. 1). From 2011 to 2030, the demand for shoulder arthroplasties is projected to increase by $333.3 \%$ (95\% CI, 257.0-432.5) for patients 55 years or younger and by 755.4\% (95\% CI, 380.7-1511.1) for patients older than 55 years. While the demand for shoulder arthroplasties in younger patients was projected to increase in our model, the demand for primary shoulder arthroplasties among patients 55 years or younger was projected to decrease to $4 \%$ (95\% CI, 2.6-5.9) of all recipients by 2030 . Therefore, while the demand for shoulder arthroplasties by younger 

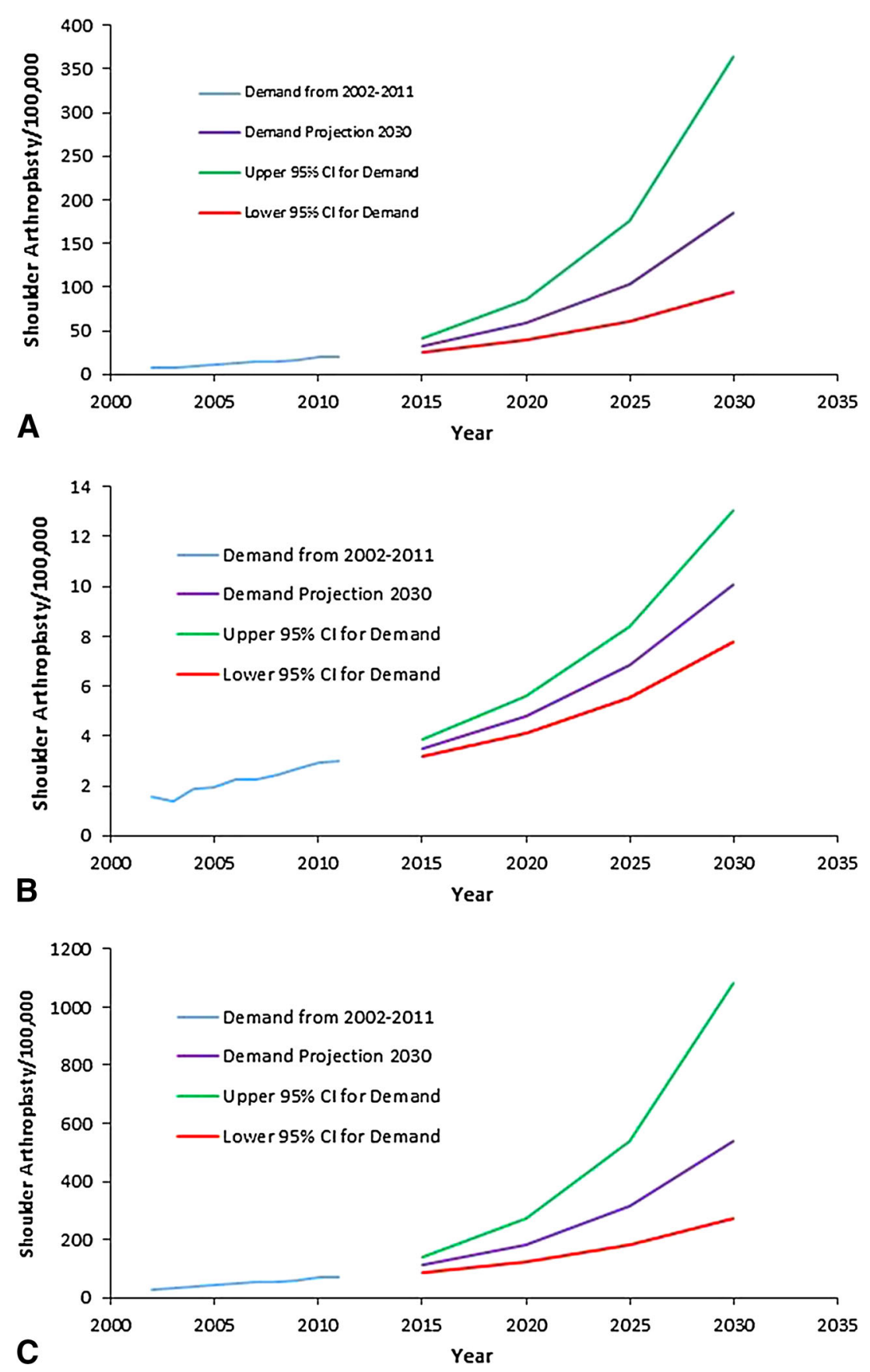

Fig. 1A-C The demand for shoulder arthroplasties for the (A) entire population, (B) the population 55 years and younger, and $(\mathbf{C})$ the arthroplasties per 100,000 people with associated $95 \%$ CIs through 2030 are shown.

Finally, our model predicts changes in the type of procedure that younger patients are likely to undergo. Rates of shoulder hemiarthroplasties in patients 55 years or younger showed a $16.5 \%$ decline per year $(95 \% \mathrm{CI}$, 16.1-7.1) from 2002 (53.6\% of all primary shoulder arthroplasties performed on patients 55 years or younger) to 2011 (34.2\% of all primary shoulder arthroplasties performed on patients 55 years or younger). By 2030, 
hemiarthroplasties are projected to account for $23.5 \%$ of all primary shoulder arthroplasties performed on patients 55 years or younger. For patients older than 55 years, hemiarthroplasties accounted for $58.4 \%$ of all arthroplasties in $2002,21.2 \%$ in 2011 , and are projected to account for $3.9 \%$ in 2030.

\section{Discussion}

The outcomes of shoulder arthroplasties in younger patients are not as reliable compared with those of the general population. Higher complication and revision rates in younger patients present direct costs to the healthcare system and indirect costs to the patient in terms of quality of life $[35,36]$. Previous studies have suggested an increased demand for shoulder arthroplasties overall [11, 19], but to our knowledge, the demand in younger patients has not been explored. Therefore, we thought that development of a projection of future shoulder arthroplasty demand in young patients would be valuable. Our model of primary shoulder arthroplasty demand, similar to a previous projection model [11], showed increased demand for shoulder arthroplasties. From our model, we project an approximately ninefold increase in the rate for shoulder arthroplasties by 2030 . Despite the predicted increase in demand for all patients and the younger subpopulation, the demand in older patients is growing at a faster rate than in younger patients. This observed trend does not follow that seen in hip and knee arthroplasties where demand in younger patients is growing faster than demand in older patients [21]. From a procedure-specific standpoint, our study showed the rates of hemiarthroplasties in younger and older patients are decreasing steadily, resulting in a greater proportion of patients undergoing anatomic TSAs or reverse shoulder arthroplasties.

Our study has several limitations. Data from the NIS have been used to study economic burden, complication rates, and future demand for hip and knee arthroplasties [21-23]; however, the scope of the NIS database creates inherent flaws that must be acknowledged. Our projections likely understate the demand for shoulder arthroplasties as ICD-9-CM code 81.83 (other repair of shoulder with arthroplasty) was excluded. Although the 81.83 code does capture some primary arthroplasty cases, our institutional experience has shown that the code number often is assigned in revision settings or nonarthroplasty cases. As noted in the Methods section, we were unable to use ICD9-CM codes in the NIS database to distinguish TSA from reverse shoulder arthroplasty before 2010; therefore, projecting future demand for reverse shoulder arthroplasty currently is not reliable based on NIS data. Additionally, we excluded revision arthroplasty cases from this study owing to the nonspecific nature of ICD-9CM coding for this procedure. Revision shoulder and elbow arthroplasties are covered by ICD-9CM code 81.97 (revision of joint replacement of upper extremity) and therefore NIS analysis that includes this ICD-9CM code would not be specific to revision shoulder arthroplasty. Another weakness is that defining an age cutoff for the younger patient is arbitrary. Selecting a younger age for this cutoff likely would reduce the projected demand for shoulder arthroplasties whereas selecting an older age would likely increase projected demands. The age of 55 years was selected because previous studies of shoulder arthroplasties in younger patients have used this age cutoff $[4,5]$. Finally, as noted by Kurtz et al. [21] in their projection study of the demand for hip or knee arthroplasties using the NIS, our model does not account for technologic advances in medicine that may reduce the need for shoulder arthroplasties, changes in public health policy and economics that may limit access to care, or the need for trained surgeons that may not meet patient demand for shoulder arthroplasties. The model also cannot account for saturation of demand in shoulder arthroplasties; it is projected based solely on the increasing demand between the years for which data have been calculated (20022011). It is impossible for demand to increase exponentially, infinitely into the future without patient demand becoming stagnant. The difficulty of accurate projection even less than two decades into the future (2030) is underscored by the broad $95 \%$ CIs for these projections. The difficulty of future projections is underscored because the data regarding demand for primary and revision knee arthroplasties presented by Kurtz et al. [21] was overprojected.

Despite these limitations, increased demand for shoulder arthroplasties was shown by our study model. Our results corroborate those seen with previous models [11, 19]. This increase in demand for shoulder arthroplasties may be a result of increased practice focus on shoulder arthroplasty [11], increased emphasis on shoulder arthroplasty in fellowship training [11], and expansion of arthroplasty indications [29]. Additionally, with the introduction of the Affordable Care Act, we expect increased access to healthcare for tens of millions of patients [39]. With this increased access to care, there may be a concomitant increase in procedural demand. Furthermore, demand for shoulder arthroplasties was projected to increase for all patients and the younger subpopulation (although at a slower rate for younger patients). The increased demand by younger patients may have implications on future surgical training and cause a drive to further surgical innovation in shoulder arthroplasties, as younger patients tend to have inconsistent results $[35,36]$. In the context of increasing demand for the difficult clinical problem of end-stage glenohumeral arthritis in younger patients, new techniques 
may be a promising area for further study. In some studies, TSA [24], humeral head resurfacing [30], and hemiarthroplasty with concentric glenoid reaming [31] in younger patients have shown promising short- and midterm results. We may see an additional increase in arthroplasty demand in younger patients with end-stage glenohumeral arthritis as arthroplasty options in this population improve. Conversely, demand in younger patients may decrease if more palatable nonarthroplasty options develop. Techniques such as arthroscopic débridement, capsular release, humeral osteoplasty and/or axillary nerve decompression [25, 27, 33], osteochondral autologous transplant [16, 32], and biologic resurfacing $[15,20,26]$ have had mixed results. Future developments in arthroplasty and nonarthroplasty treatment options for end-stage glenohumeral arthritis in young people will shape future demand up to and beyond the window of this predictive model.

The changing demographics of patients undergoing shoulder arthroplasties observed in our study differs from changes observed in the patient population for hip and knee arthroplasties. In shoulder and hip and knee arthroplasties, procedural demand for younger and older patients is increasing [11, 19, 21]. However, our model predicts that demand is increasing for younger patients at a rate slower than that of the general population while the converse has been found for hip and knee arthroplasties [21]. Younger patients are projected to account for more than $1 / 2$ the volume of all patients undergoing primary hip and knee arthroplasties [21], while patients younger than 55 years are predicted to account for only $4 \%$ of patients undergoing shoulder arthroplasties by $2030 \mathrm{in}$ this model. These findings may result from numerous factors. First, a change in population demographics may be contributory. According to the United States Census Bureau data, the percentage of people 55 years or younger in 2011 account for $74.5 \%$. This is projected to decrease to $68.9 \%$ in 2030. This decrease in the overall percentage of young people may be contributing to the observed change in demographic trends. Second, the volume increase attributable to the popularity of the reverse prosthesis would disproportionately affect the elderly patient population, as reverse prostheses usually are not implanted in younger patients. The Administration on Aging reported that citizens older than 65 years represented $12.4 \%$ of the population in $2000,12.9 \%$ in 2009 , and projected that the population will increase to $19 \%$ by 2030 [2]. Market analysis found approximately 2000 reverse total shoulder arthroplasties performed in 2004, 10,000 in 2007, and a projected volume of 30,000 in 2012 [19]. We were unable to clearly elucidate demand for reverse shoulder arthroplasties from the NIS data owing to previously noted confounding procedural codes, but a decreased number of hemiarthroplasties in the face of rising demand for primary shoulder arthroplasties suggests wider use of reverse shoulder arthroplasties and TSAs. With reverse shoulder arthroplasty having an ICD-9-CM code in recent years, further study may reveal if the increase in shoulder arthroplasties is being carried by reverse shoulder arthroplasties. The increasing demand for reverse shoulder arthroplasties will be able to be more thoroughly evaluated when the coding is used more universally and as patients and surgeons become more comfortable with this procedure. We anticipate that the demand may increase as patients waiting to see longer-term results become comfortable enough to commit to this procedure. Third, outcomes in younger patients undergoing shoulder arthroplasties have been poorer than those for the general population [35, 36], whereas outcomes of hip and knee arthroplasties in younger patients have been studied extensively and show promising results at 10 and 15 years followup [8, 10, 13, 18, 28].

Our study also projects decreasing use of hemiarthroplasties compared with TSAs in younger patients. Studies showing that TSAs yield superior clinical results and lower revision rates compared with hemiarthroplasties in younger patients may explain these projections [5,41]. Although data suggest greater survivorship of TSAs in younger patients compared with hemiarthroplasties [35, 36], a previous study suggested concerning rates of early glenoid loosening [12]. The implications of the observed trend of decreasing hemiarthroplasties in younger patients on the revision arthroplasty burden is yet unknown. The observed trend and projection of decreasing use of hemiarthroplasties in older patients are not surprising. Analyses have shown poorer long-term outcomes with hemiarthroplasty compared with TSA for older patients with glenohumeral joint arthritis [3, $14,37,40,41]$. Additionally, the expansion of indications for reverse shoulder arthroplasties, specifically for patients with proximal humerus fractures $[1,6,7,34]$ previously treated almost exclusively with hemiarthroplasties, may explain the decline in hemiarthroplasties in treating elderly patients. The decline may accelerate as some comparative results between reverse shoulder arthroplasty and hemiarthroplasty have shown superiority of the reverse prosthesis in patients with complex proximal humerus fractures [9] and in patients with rotator cuff insufficiency [41].

We predict continued increased demand for primary shoulder arthroplasties. The projected increase in demand has important implications for the future of revision arthroplasty, including costs to the healthcare system and need for qualified surgeons to meet the demand. Despite the growth rate for younger patients, current and projected demands remain greatest in the older patient population. The discrepancy may be related to changing indications, poorer reported outcomes in younger patients, the evolution of nonarthroplasty options for younger patients, and the recent increased popularity of reverse shoulder arthroplasties in older patients (specifically, for displaced proximal humerus fractures). 


\section{References}

1. Acevedo DC, VanBeek C, Lazarus MD, Williams GR, Abboud JA. Reverse shoulder arthroplasty for proximal humeral fractures: update on indications, technique, and results. J Shoulder Elbow Surg. 2014;23:279-289.

2. ACL Administration for Community Living. Administration on Aging (AoA). Projected Future Growth of the Older Population. Available at: http://www.aoa.gov/Aging_Statistics/. Accessed June 30, 2014.

3. Adams JE, Sperling JW, Schleck CD, Harmsen WS, Cofield RH. Outcomes of shoulder arthroplasty in Olmsted County, Minnesota: a population-based study. Clin Orthop Relat Res. 2007; 455:176-182.

4. Bailie DS, Llinas PJ, Ellenbecker TS. Cementless humeral resurfacing arthroplasty in active patients less than fifty-five years of age. J Bone Joint Surg Am. 2008;90:110-117.

5. Bartelt R, Sperling JW, Schleck CD, Cofield RH. Shoulder arthroplasty in patients aged fifty-five years or younger with osteoarthritis. J Shoulder Elbow. Surg. 2011;20:123-130.

6. Bufquin T, Hersan A, Hubert L, Massin P. Reverse shoulder arthroplasty for the treatment of three- and four-part fractures of the proximal humerus in the elderly: a prospective review of 43 cases with a short-term follow-up. J Bone Joint Surg Br. 2007;89:516-520.

7. Cazeneuve JF, Cristofari DJ. Grammont reversed prosthesis for acute complex fracture of the proximal humerus in an elderly population with 5 to 12 years follow-up. Orthop Traumatol Surg Res. 2014;100:93-97.

8. Chiu KY, Tang WM, Ng TP, Poon KC, Ho WY, Lee KM. Cementless total hip arthroplasty in young Chinese patients: a comparison of 2 different prostheses. J Arthroplasty. 2001;16: 863-870.

9. Cuff DJ, Pupello DR. Comparison of hemiarthroplasty and reverse shoulder arthroplasty for the treatment of proximal humeral fractures in elderly patients. J Bone Joint Surg Am. 2013;95: 2050-2055.

10. Dalury DF, Ewald FC, Christie MJ, Scott RD. Total knee arthroplasty in a group of patients less than 45 years of age. J Arthroplasty. 1995;10:598-602.

11. Day JS, Lau E, Ong KL, Williams GR, Ramsey ML, Kurtz SM. Prevalence and projections of total shoulder and elbow arthroplasty in the United States to 2015. J Shoulder Elbow Surg. 2010;19:1115-1120.

12. Denard PJ, Raiss P, Sowa B, Walch G. Mid- to long-term followup of total shoulder arthroplasty using a keeled glenoid in young adults with primary glenohumeral arthritis. J Shoulder Elbow Surg. 2013;22:894-900.

13. Diduch DR, Insall JN, Scott WN, Scuderi GR, Font-Rodriguez D. Total knee replacement in young, active patients: long-term follow-up and functional outcome. J Bone Joint Surg Am. 1997; 79:575-582.

14. Duan X, Zhang W, Dong X, Liu M, Gao Y, Huang F, Li J, Xiang $Z$. Total shoulder arthroplasty versus hemiarthroplasty in patients with shoulder osteoarthritis: a meta-analysis of randomized controlled trials. Semin Arthritis Rheum. 2013;43:297302.

15. Elhassan B, Ozbaydar M, Diller D, Higgins LD, Warner JJ. Softtissue resurfacing of the glenoid in the treatment of glenohumeral arthritis in active patients less than fifty years old. J Bone Joint Surg Am. 2009;91:419-424.

16. Gobezie R, Lenarz CJ, Wanner JP, Streit JJ. All-arthroscopic biologic total shoulder resurfacing. Arthroscopy. 2011;27:1588-1593.

17. Healthcare Cost and Utilization Project (HCUP). Introduction to the HCUP National Inpatient Sample (NIS) 2012. Agency for
Healthcare Research and Quality; 2014. Available at: http:// www.hcup-us.ahrq.gov/nisoverview.jsp. Accessed July 16, 2014.

18. Hofmann AA, Heithoff SM, Camargo M. Cementless total knee arthroplasty in patients 50 years or younger. Clin Orthop Relat Res. 2002;404:102-107.

19. Kim SH, Wise BL, Zhang Y, Szabo RM. Increasing incidence of shoulder arthroplasty in the United States. J Bone Joint Surg Am. 2011;93:2249-2254.

20. Krishnan SG. Humeral hemiarthroplasty with biologic resurfacing of the glenoid for glenohumeral arthritis: two to fifteen-year outcomes. J Bone Joint Surg Am. 2007;89:727-734.

21. Kurtz SM, Lau E, Ong K, Zhao K, Kelly M, Bozic KJ. Future young patient demand for primary and revision joint replacement: national projections from 2010 to 2030. Clin Orthop Relat Res. 2009;467:2606-2612.

22. Kurtz SM, Lau E, Schmier J, Ong KL, Zhao K, Parvizi J. Infection burden for hip and knee arthroplasty in the United States. J Arthroplasty. 2008;23:984-991.

23. Kurtz SM, Lau E, Watson H, Schmier JK, Parvizi J. Economic burden of periprosthetic joint infection in the United States. $J$ Arthroplasty. 2012;27(8 suppl):61-65.e1.

24. Levy JC, Virani NA, Frankle MA, Cuff D, Pupello DR, Hamelin JA. Young patients with shoulder chondrolysis following arthroscopic shoulder surgery treated with total shoulder arthroplasty. J Shoulder Elbow Surg. 2008;17:380-388.

25. Millett PJ, Gaskill TR. Arthroscopic trans-capsular axillary nerve decompression: indication and surgical technique. Arthroscopy. 2011;27:1444-1448.

26. Namdari S, Alosh H, Baldwin K, Glaser D, Kelly JD. Biological glenoid resurfacing for glenohumeral osteoarthritis: a systematic review. J Shoulder Elbow Surg. 2011;20:1184-1190.

27. Namdari S, Skelley N, Keener JD, Galatz LM, Yamaguchi K. What is the role of arthroscopic debridement for glenohumeral arthritis? A critical examination of the literature. Arthroscopy. 2013;29:1392-1398.

28. Nercessian OA, Wu WH, Sarkissian H. Clinical and radiographic results of cementless AML total hip arthroplasty in young patients. J Arthroplasty. 2001;16:312-316.

29. Ponce BA, Oladeji LO, Rogers ME, Menendez ME. Comparative analysis of anatomic and reverse total shoulder arthroplasty: inhospital outcomes and costs. J Shoulder Elbow Surg. 2015;24: 460-467.

30. Raiss P, Aldinger PR, Kasten P, Rickert M, Loew M. Total shoulder replacement in young and middle-aged patients with glenohumeral osteoarthritis. J Bone Joint Surg Br. 2008;90:764-769.

31. Saltzman MD, Chamberlain AM, Mercer DM, Warme WJ, Bertelsen AL, Matsen FA. Shoulder hemiarthroplasty with concentric glenoid reaming in patients 55 years old or less. $J$ Shoulder Elbow Surg. 2011;20:609-615.

32. Scheibel M, Bartl C, Magosch P, Lichtenberg S, Habermeyer P. Osteochondral autologous transplantation for the treatment of full-thickness articular cartilage defects of the shoulder. $J$ Bone Joint Surg Br. 2004;86:991-997.

33. Skelley NW, Namdari S, Chamberlain AM, Keener JD, Galatz LM, Yamaguchi K. Arthroscopic debridement and capsular release for the treatment of shoulder osteoarthritis. Arthroscopy. 2014 Nov 8. [Epub ahead of print].

34. Smith CD, Guyver P, Bunker TD. Indications for reverse shoulder replacement: a systematic review. J Bone Joint Surg Br. 2012;94:577-583

35. Sperling JW, Cofield RH, Rowland CM. Neer hemiarthroplasty and Neer total shoulder arthroplasty in patients fifty years old or less: long-term results. J Bone Joint Surg Am. 1998;80:464-473.

36. Sperling JW, Cofield RH, Rowland CM. Minimum fifteen-year follow-up of Neer hemiarthroplasty and total shoulder 
arthroplasty in patients aged fifty years or younger. $J$ Shoulder Elbow Surg. 2004;13:604-613.

37. Sperling JW, Cofield RH, Schleck CD, Harmsen WS. Total shoulder arthroplasty versus hemiarthroplasty for rheumatoid arthritis of the shoulder: results of 303 consecutive cases. $J$ Shoulder Elbow Surg. 2007;16:683-690.

38. United States Census Bureau. National Characteristics: Vintage 2013 - U.S Census Bureau. Available at: http://www.census.gov/ popest/data/national/asrh/2013/index.html. Accessed January 16, 2015.

39. United States Congress. Public Law 111 - 148 - Patient Protection and Affordable Care Act. Available at: http://www.gpo.gov/
fdsys/pkg/PLAW-111publ148/content-detail.html. Accessed July 16, 2014.

40. van den Bekerom MP, Geervliet PC, Somford MP, van den Borne MP, Boer R. Total shoulder arthroplasty versus hemiarthroplasty for glenohumeral arthritis: a systematic review of the literature at long-term follow-up. Int J Shoulder Surg. 2013;7:110-115.

41. Young SW, Zhu M, Walker CG, Poon PC. Comparison of functional outcomes of reverse shoulder arthroplasty with those of hemiarthroplasty in the treatment of cuff-tear arthropathy: a matched-pair analysis. J Bone Joint Surg Am. 2013;95:910-915. 